Research in Astron. Astrophys. 2020 Vol. X No. XX, 000-000

http://www.raa-journal.org http://www.iop.org/journals/raa

$\boldsymbol{R}$ esearch in

(LTEX: 2020-0126.tex; printed on August 5, 2020; 4:10)

Astronomy and

Astrophysics

\title{
He-shell flashes on the surface of oxygen-neon white dwarfs
}

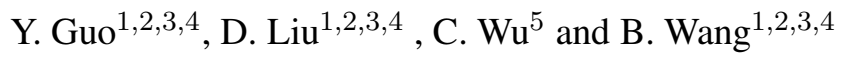 \\ 1 Yunnan Observatories, Chinese Academy of Sciences, Kunming 650216, China; \\ liudongdong@ynao.ac.cn;wangbo@ynao.ac.cn \\ 2 Key Laboratory for the Structure and Evolution of Celestial Objects, Chinese Academy of Sciences, \\ Kunming 650216, China \\ 3 University of Chinese Academy of Sciences, Beijing 100049, China \\ 4 Center for Astronomical Mega-Science, Chinese Academy of Sciences, Beijing 100012, China \\ 5 Physics Department and Tsinghua Center for Astrophysics (THCA), Tsinghua University, Beijing \\ 100084, China
}

\begin{abstract}
Accretion induced collapse (AIC) may be responsible for the formation of some interesting neutron star binaries, e.g., millisecond pulsars, intermediate-mass binary pulsars, etc. It has been suggested that oxygen-neon white dwarfs (ONe WDs) can increase their mass to the Chandrasekhar limit by multiple He-shell flashes, leading to AIC events. However, the properties of He-shell flashes on the surface of ONe WDs are still not well understood. In this article, we aim to study He-shell flashes on the surface of ONe WDs in a systematic approach. We investigated the long-term evolution of ONe WDs accreting He-rich material with various constant mass-accretion rates by time-dependent calculations with the stellar evolution code Modules for Experiments in Stellar Astrophysics (MESA), in which the initial ONe WD masses range from 1.1 to $1.35 M_{\odot}$. We found that the mass-retention efficiency increases with the ONe WD mass and the mass-accretion rate, whereas both the nova cycle duration and the ignition mass decrease with the $\mathrm{ONe}$ WD mass and the mass-accretion rate. We also present the nuclear products in different accretion scenarios. The results presented in this article can be used in the future binary population synthesis studies of AIC events.
\end{abstract}

Key words: stars: evolution — binaries: close — supernovae: general — white dwarfs

\section{INTRODUCTION}

It has been widely accepted that carbon-oxygen white dwarfs (CO WDs) can form type Ia supernovae when they grow in mass close to the Chandrasekhar limit $\left(M_{\mathrm{Ch}}\right)$ (e.g., Nomoto et al. 1997 , Hillebrandt \& Niemeyer 2000). Nevertheless, oxygen-neon white dwarfs (ONe WDs) may undergo accretion-induced collapse (AIC) when their mass approach $M_{\mathrm{Ch}}$, forming neutron stars (NSs) eventually (e.g., Miyaji et al. 1980; Nomoto 1984; Nomoto \& Kondo 1991; Zha et al. 2019). AIC events are predicted to be very faint and fast transients, which have not been identified directly so far (e.g., Woosley \& Baron 1992; Dessart et al. 2006). However, it is notable that AIC process can form some interesting objects in the observations, such as X-ray binaries, millisecond pulsars and low-/intermediatemass binary pulsars (e.g., Bailvn \& Grindlav 1990; Bhattacharya \& van den Heuvel 1991; Usov 1992; Podsiadlowski et al. 2002; Tauris et al. 2013; Ablimit \& Li 2015; Liu \& Li 2017; Liu et al. 2018). It has been suggested that AIC process may also relate to the r-process nucleosynthesis, the formation of rapidly spinning magnetars and ultra-high-energy cosmic rays like gamma-ray bursts (e.g., 
Wheeler et al. 1998; Frver et al. 1999; Oian \& Wasserburg 2007; Dar et al. 1992; Usov 1992; Moriya 2016; Piro \& Kollmeier 2016; Lyutikov \& Toonen 2017). Furthermore, AIC is a potential source of gravitational wave emission (e.g., Abdikamalov et al. 2010). For a recent review on the AIC of ONe WDs, see Wang \& Liu (2020).

Although the AIC process is a non-negligible channel to form NSs, their progenitor model is still unclear. There are two classic progenitor models of AIC events, that is, the single-degenerate (SD) model and the double-degenerate (DD) model. In the SD model, an ONe WD accretes H/He-rich material from a non-degenerate companion. The companion can be a main-sequence (MS) star, a red giant (RG) star or a He star (e.g., Canal et al. 1990; Nomoto \& Kondo 1991; Tauris et al. 2013; Brooks et al. 2017b; Liu et al. 2018; Wang 2018b). The accreted material will burn into He first, and then into $\mathrm{C}$ and $\mathrm{O}$, and then $\mathrm{Ne}$ for $\mathrm{H}$-accreting WDs, or burn into $\mathrm{C}$ and $\mathrm{O}$ first, and then $\mathrm{Ne}$ for He-accreting WDs. If ONe WD can increase its mass close to $M_{\mathrm{Ch}}$, it will collapse into a NS. Note that the final fate of accreting ONe WDs would not be affected by Urca-process (e.g., Schwab et al. 2017). Recent studies suggested that He accretion onto CO WDs may also form NSs owing to the off-center carbon ignition when the mass-accretion rate is above a critical value (see Brooks et al. 2016; Wang et al. 2017). The DD model involves the merger of two WDs shrinkage of the orbit induced by the gravitational wave radiation, including the merging of double CO WDs, double ONe WDs, or the merging of an ONe WD with a CO WD (e.g., Nomoto \& Iben 1985; Saio \& Nomoto 2004; Schwab et al. 2016; Liu \& Wang 2020).

It has been proposed that ONe WD+He star systems could experience AIC and then be recycled to form binary pulsars (e.g., Tauris et al. 2013). Wang (2018b) suggested that the ONe WD+He star channel is a dominant channel to produce AIC events in the SD model, with rates of $0.1-0.7 \times 10^{-3} \mathrm{yr}^{-1}$ and their delay times range from 30 to $180 \mathrm{Myr}$. In addition, it has been suggested that the $\mathrm{ONe} \mathrm{WD}+\mathrm{He}$ star channel may be responsible for the formation of observed intermediate-mass binary pulsars with short orbital periods (Liu et al.2018). Moreover, PSR J1802-2124 is a binary containing a $1.24 \pm 0.11 M_{\odot} \mathrm{NS}$ and a $0.78 \pm 0.04 M_{\odot} \mathrm{CO}$ WD companion with an orbital period of $0.7 \mathrm{~d}$ and a spin period of $1.2 \mathrm{~ms}$, which was first detected by the Parkes Multibeam Pulsar Survey (Faulkner et al. 2004). Liu et al. (2018) suggested that a $1.3 M_{\odot}$ ONe WD and a $1.0 M_{\odot}$ He star with an orbital period of $0.47 \mathrm{~d}$ is a possible channel to form systems like PSR J1802-2124. Note that NS+He star systems may also contribute to some of intermediate-mass binary pulsars with short orbital periods (e.g., Chen \& Liu 2013).

He-accreting ONe WDs may also relate to the formation of some other peculiar observed objects, such as AM Canum venaticorum (AM CVn) binaries and He novae. AM CVn consists of a CO/ONe WD and a He WD with accretion rates ranging from $10^{-13}$ to $10^{-5} M_{\odot} \mathrm{yr}^{-1}$ and extreme short orbital periods in the range of 5-65 min (e.g., Nelemans et al. 2001; Shen \& Bildsten 2009; Brooks et al. 2015). There are some WD+He star systems in the observations, e.g., CD-30 11223 , KPD 1930+2752, V445 Puppis, and HD 49798 with its compact companion (e.g., Geier et al. 2007; Kato et al. 2008; Woudt et al. 2009; Wang \& Han 2010; Liu et al. 2015; Wang et al. 2015; Wang 2018a). In these binaries, V445 Puppis is the only He nova that has been identified in late 2000 by Kanatsu (e.g., Kato et al. 2000).

It has been thought that the mass-accretion process plays an important role in studying binary evolution and the final fate of accreting WD (e.g., Nomoto 1982; Piersanti et al. 2014; Wang et al. 2015). There exist three regions for different mass-accretion rates: at high accretion rates, the WD will become a He-giant-like star since the accreted He-rich matter piles up on the surface of the WD (e.g., Nomoto 1982); at low accretion rates, He-shell flashes are triggered due to the unsteady He burning on the surface of the WD (e.g., Piersanti et al. 2014; Hillman et al. 2016); the accretion rates for steady He-shell burning are limited to a narrow regions (see Nomoto 1982; Wang et al. 2015). The accretion rate that can trigger He-shell flashes is generally considered to be less than $10^{-6} M_{\odot} \mathrm{yr}^{-1}$ (e.g., Kato \& Hachisu 2004: Wang et al. 2015).

The mass-retention efficiency during He-shell flashes has a great influence on binary evolution, and is defined as the ratio of the processed matter remaining after one cycle of He-shell flash to the accreted mass (e.g., Kato \& Hachisu 2004). Previous studies mainly focus on the He-shell flashes on the surface of CO WDs (e.g., Kato \& Hachisu 1999, 2004; Wang et al. 2015; Wu et al. 2017). In this work, we aim to investigate multicycle He-shell flashes on the surface of ONe WDs and provide some features 
of He-accreting ONe WDs, such as the mass-retention efficiencies, nova cycle durations and ignition masses with different accretion rates for various ONe WD masses. We also present the nuclear products in different accretion scenarios. In Sect. 2, we show the basic assumptions and methods in our numerical calculations. We show the numerical results in Sect. 3. Finally, we present discussions and summary in Sect. 4 .

\section{METHODS}

We use the stellar evolution code Modules for Experiments in Stellar Astrophysics (MESA, version 10398; see Paxton et al. 2011, 2013, 2015, 2018) to simulate the long-term evolution of He-acrreting ONe WDs. Using the suite case make_o_ne_wd in MESA, we evolve pre-MS stars with different masses to the WD cooling phase, and finally obtain ONe WDs with masses of $1.1,1.2,1.3,1.35 M_{\odot}$. The suite case $\mathrm{wd} 2$ is used to calculate the accretion WDs and the mass ejection processes during nova outbursts. The mass-accretion rates are assumed in the range of $3 \times 10^{-7}-2 \times 10^{-6} M_{\odot} \mathrm{yr}^{-1}$, for which the WDs would undergo multicycle He-shell flashes. We set the mass fraction of $\mathrm{He}$ and metallicity in the accreted material to be 0.98 and 0.02 , respectively.

The MESA nuclear reaction network co_burn.net is adopted in our calculations, including helium, carbon, oxygen burning coupled by 57 reactions (e.g., ${ }^{3} \mathbf{H e},{ }^{4} \mathbf{H e},{ }^{7} \mathbf{L i},{ }^{7} \mathbf{B e}$, $\left.{ }^{8} \mathbf{B},{ }^{12} \mathbf{C},{ }^{14} \mathbf{N},{ }^{15} \mathrm{~N},{ }^{16} \mathrm{O},{ }^{19} \mathbf{F},{ }^{20} \mathrm{Ne},{ }^{23} \mathrm{Na},{ }^{24} \mathrm{Mg},{ }^{27} \mathrm{Al},{ }^{28} \mathrm{Si}\right)$. This nuclear reaction network mainly includes reactions as follows:

$$
\begin{gathered}
{ }^{4} \mathrm{He}+{ }^{4} \mathrm{He}+{ }^{4} \mathrm{He} \rightarrow{ }^{12} \mathbf{C}+\gamma, \\
{ }^{12} \mathbf{H e} \rightarrow{ }^{16} \mathbf{O}+\gamma, \\
{ }^{16} \mathbf{C} \rightarrow{ }^{20} \mathbf{O}+{ }^{4} \mathbf{H e} \rightarrow{ }^{4} \mathbf{H e}, \\
{ }^{20} \mathbf{N e}+\gamma, \\
{ }^{20} \mathbf{N e}+{ }^{4} \mathbf{H e} \rightarrow{ }^{24} \mathbf{M g}+\gamma, \\
{ }^{12} \mathbf{C}+{ }^{16} \mathbf{O} \rightarrow{ }^{28} \mathbf{S i},
\end{gathered}
$$

In our simulation, the default OPAL opacity is adopted, which is applicable to extra carbon and oxygen during the He-shell flash (e.g., Iglesias \& Rogers 1996). We set the factor that determines the optical depth $(\tau)$ of the outer edge of the stellar model to be 50, i.e., assuming that $\tau$ at the boundary of the stellar model is $50 \times 2 / 3$. In addition, the value of mixing-length parameter is set to 2.0 , which is the ratio of the mixing length to the pressure scaleheight. Furthermore, we do not consider the influence of hydrodynamics, rotation and convective overshooting in this work.

In the present work, the super-Eddington wind is adopted when ONe WDs undergo multicycle Heshell flashes. The Eddington luminosity $\left(L_{\mathrm{Edd}}\right)$ and the wind mass-loss rate $(\dot{M})$ can be expressed as follows:

$$
\begin{gathered}
L_{\mathrm{Edd}}=\frac{4 \pi G c M_{\mathrm{WD}}}{\tau}, \\
\dot{M}=\frac{2 \eta_{\mathrm{Edd}}\left(L_{\mathrm{eff}}-L_{\mathrm{Edd}}\right)}{v_{\mathrm{esc}}^{2}},
\end{gathered}
$$

where $c, \tau, G, v_{\text {esc }}$ are the vacuum speed of light, Rosseland mean opacity, gravitational constant and the escape velocity at the photosphere of accreting ONe WDs, respectively. We assume that all energy above the Eddington luminosity is used to eject matter (i.e., the super-Eddington wind factor $\eta_{\mathrm{Edd}}=1$ ). 


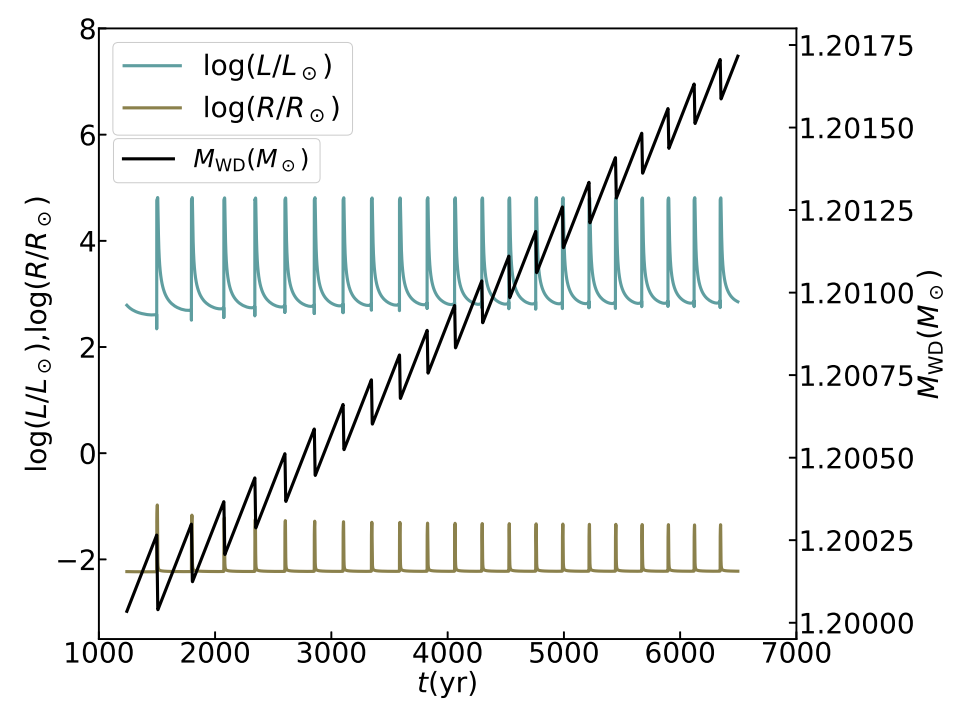

Fig. 1: A representative example of multicycle He-shell flashes on the surface of a $1.2 M_{\odot}$ ONe WD, in which we set the mass-accretion rate $\left(\dot{M}_{\text {acc }}\right)$ to be $9 \times 10^{-7} M_{\odot} \mathrm{yr}^{-1}$.

In this case, some of the accumulated material will be blown away when the super-Eddington wind is triggered. The mass-retention efficiency $\left(\eta_{\mathrm{He}}\right)$ can be expressed as follow:

$$
\eta_{\mathrm{He}}=\frac{M_{\mathrm{acc}}-M_{\mathrm{ej}}}{M_{\mathrm{acc}}}
$$

where $M_{\text {acc }}$ is the mass accreted by a ONe WD during a single nova cycle (i.e., from the end of one outburst to the end of the next one) and $M_{\mathrm{ej}}$ is the ejected mass. In the present work, we simulate about 20 of successive He-shell flashes and provide the average mass-retention efficiency in the results.

\section{NUMERICAL RESULTS}

\subsection{An example of He-shell flashes}

The He-shell accreted onto the surface of ONe WDs are expected to be ignited and start to burn when its mass reaches a critical value. Fig. 1 shows the long-term evolution of the radius, mass and luminosity of a 1.2 $M_{\odot}$ ONe WD accreting He-rich material at the mass-accretion rate of $9 \times 10^{-7} M_{\odot} \mathrm{yr}^{-1}$. From this figure, we can see that some of the accreted material is blown away during He-shell flashes, and the remaining material leads to the increase of the WD mass. In this case, the mass of ONe WD would increase to $M_{\mathrm{Ch}}$ after much longer evolution, which may lead to the occurrence of the electron-capture reactions of ${ }^{24} \mathrm{Mg}\left({ }^{24} \mathrm{Mg} \rightarrow{ }^{24} \mathrm{Ne}\right)$ and ${ }^{20} \mathrm{Ne}\left({ }^{20} \mathrm{Ne} \rightarrow{ }^{20} \mathrm{O}\right.$ ) (e.g., Miyaji et al. 1980; Nomoto \& Kondo 1991; Schwab et al. 2015; Wu \& Wang 2018). In addition, we can also see the He shell outburst periodically. Especially, the He-shell outbursts tend to become milder as the surface of ONe WD is heated up by the first flash.

Fig. 2 presents the Hertzsprung-Russell diagram of one cycle of He-shell flashes, in which $M_{\mathrm{WD}}=1.2 M_{\odot}$ and $\dot{M}_{\mathrm{acc}}=9 \times 10^{-7} M_{\odot} \mathrm{yr}^{-1}$. Important phases during the evolution are marked in this figure. $\mathrm{A} \rightarrow \mathrm{B}$ : ONe WD accretes He-rich material, during which the effective temperature $T_{\text {eff }}$ and the luminosity $L$ decrease since almost no He-shell burning in this stage to provide the energy to balance the radiative losses; $\mathrm{B} \rightarrow \mathrm{C}$ : the convective shell begin to attain the surface of $\mathrm{ONe}$ WD gradually; 


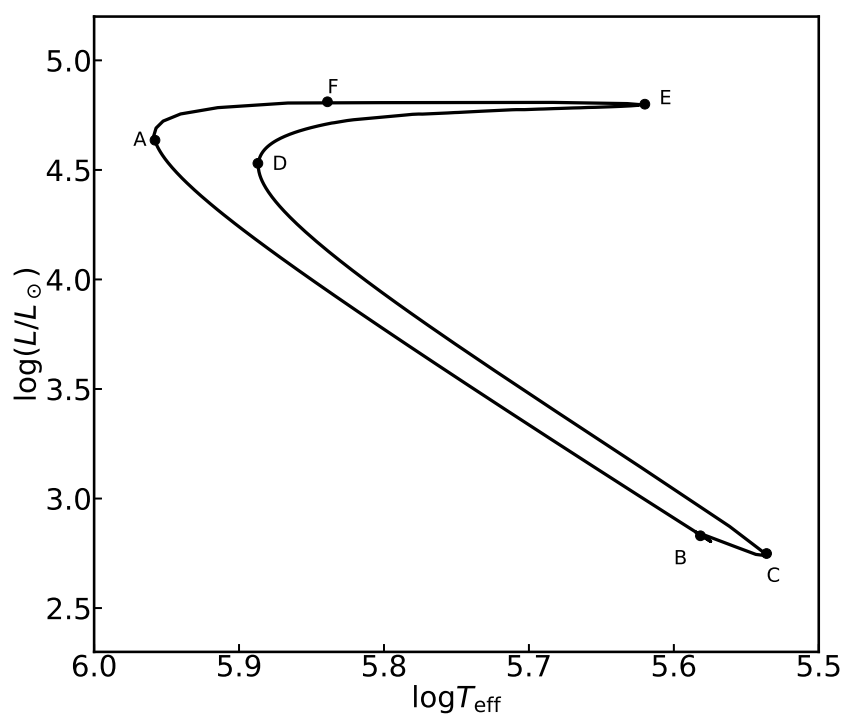

Fig. 2: Hertzsprung-Russell diagram showing a single cycle He-shell flash, with $M_{\mathrm{WD}}$ and $\dot{M}_{\mathrm{acc}}$ as $1.2 M_{\odot}$ and $9 \times 10^{-7} M_{\odot} \mathrm{yr}^{-1}$, respectively.

$\mathrm{C} \rightarrow \mathrm{D}: T_{\text {eff }}$ and $L$ increase rapidly since the He-shell flash turns into a thermonuclear runaway; $\mathrm{D} \rightarrow \mathrm{E}$ : after the luminosity of ONe WD exceeds $L_{\mathrm{Edd}}$, the radius of ONe WD will expand and cause mass loss. The radius of $\mathrm{ONe}$ WD reaches its maximum value at point $\mathrm{E}$; $\mathrm{E} \rightarrow \mathrm{F}$ : radius contraction makes a main contribution to provide energy for mass ejection. Meanwhile, $T_{\text {eff }}$ increases and reaches point $\mathrm{F}$ where the wind mass loss stops (see also Kato \& Hachisu 1994; Chen et al. 2019).

\subsection{He-shell flashes in different accretion scenarios}

Fig. 3 shows the mass-retention efficiencies $\left(\eta_{\mathrm{He}}\right)$, nova cycle durations $(D)$ and ignition masses $\left(M_{\text {ign }}\right)$ changing with the accretion rates for different ONe WD masses. Panel (a) presents the mass-retention efficiencies during He-shell flashes throughout the entire $\left(M_{\mathrm{WD}}, \dot{M}_{\mathrm{acc}}\right)$ grid. From this figure, we can see that the mass-retention efficiency increases with the mass-accretion rate for a given ONe WD mass. This is because the degeneracy of He-shell is lower at higher accretion rates, resulting in weaker flashes of the He shell and thus less material to be blown away. When the mass-accretion rate is close to the lower boundary of the helium steady burning region, the mass-retention efficiency would approach to 1 , which means that almost all of the material remains on the surface of ONe WD. It is also notable that the mass-retention efficiency increases with the ONe WD mass for a given accretion rate. This is because more massive WD have higher surface gravitation, resulting in nuclear burning rates much higher than wind mass loss rates. Therefore, we can infer that more material could accumulate on the surface of more massive $\mathrm{ONe}$ WDs for a given mass-accretion rate.

The cycle duration is an important observable feature for recurrent novae, which is defined as the time interval between two successive He-shell flashes (e.g., Hillman et al. 2015, 2016). Panel (b) shows the nova cycle durations for different mass-accretion rates and ONe WD masses. Obviously, the duration decreases with the mass-accretion rate for a given ONe WD mass, and also decreases with the WD mass for a given accretion rate. This is because the He-shell mass required for nova outburst is smaller for massive ONe WDs due to their higher surface gravitation, as shown in panel (c). For a given ONe WD, 

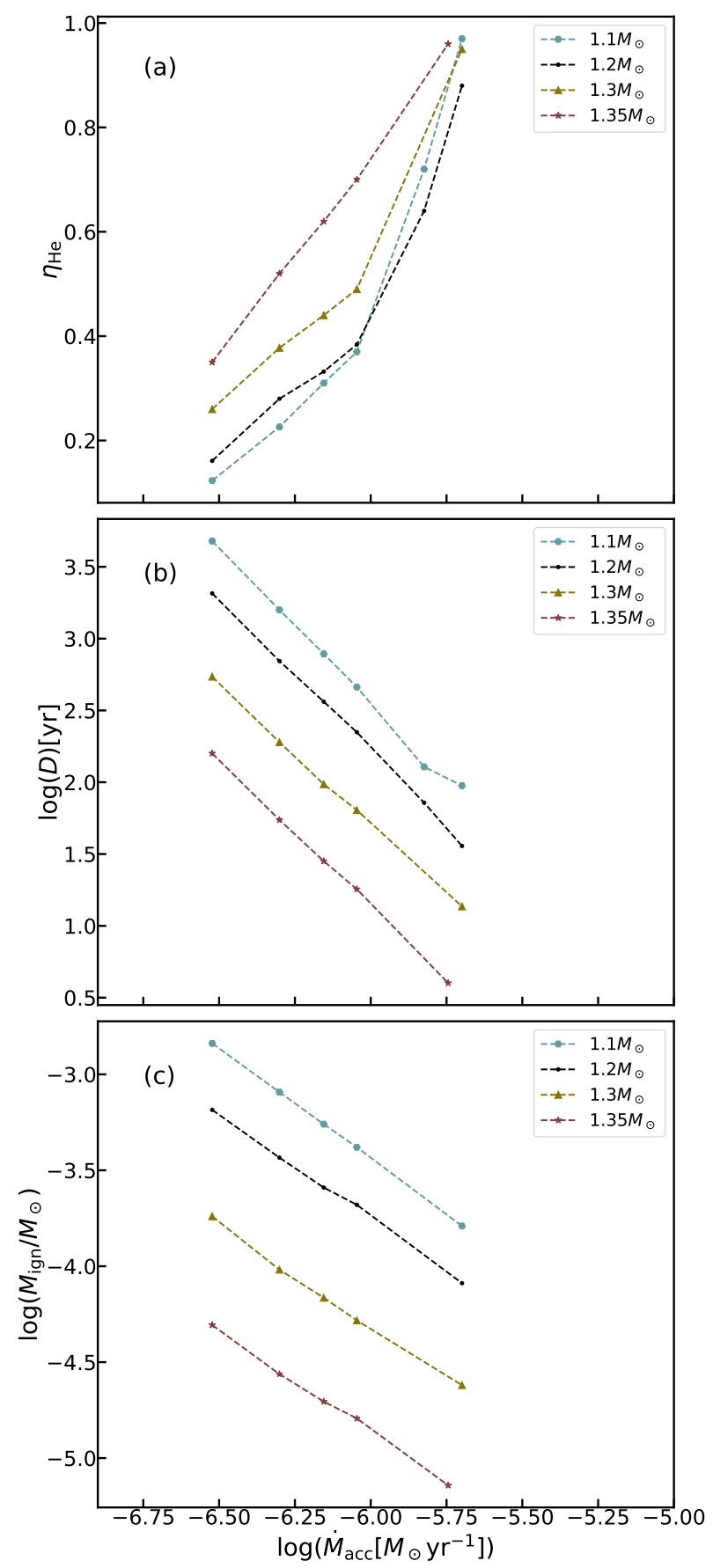

Fig. 3: Panel (a), (b) and (c) show the mass-retention efficiency $\eta_{\mathrm{He}}$, nova cycle duration $D$, and ignition mass $M_{\text {ign }}$ changing with the accretion rate $\dot{M}_{\text {acc }}$ for different ONe WD masses, respectively. 
Table 1: Properties of He-shell flashes without mixing.

\begin{tabular}{|c|c|c|c|c|c|}
\hline $\begin{array}{l}M_{\mathrm{WD}} \\
\left(M_{\odot}\right) \\
\end{array}$ & $\begin{array}{c}\dot{M}_{\mathrm{acc}} \\
\left(M_{\left.\odot \mathrm{yr}^{-1}\right)}\right.\end{array}$ & $\begin{array}{c}M_{\text {ign }} \\
\left(M_{\odot}\right) \\
\end{array}$ & $\begin{array}{c}M_{\mathrm{ej}} \\
\left(M_{\odot}\right) \\
\end{array}$ & $\begin{array}{c}\log (D) \\
(\mathrm{yr})\end{array}$ & $\eta_{\mathrm{He}}$ \\
\hline 1.1 & $3 e-7$ & $1.45 \mathrm{e}-3$ & $1.28 \mathrm{e}-3$ & 3.69 & 0.12 \\
\hline 1.1 & $5 e-7$ & $8.08 \mathrm{e}-4$ & $6.26 \mathrm{e}-4$ & 3.20 & 0.23 \\
\hline 1.1 & $7 e-7$ & $5.50 \mathrm{e}-4$ & $3.76 \mathrm{e}-4$ & 2.90 & 0.31 \\
\hline 1.1 & $9 e-7$ & $4.17 \mathrm{e}-4$ & $2.65 \mathrm{e}-4$ & 2.66 & 0.37 \\
\hline 1.1 & $1.5 \mathrm{e}-6$ & $2.06 \mathrm{e}-4$ & $8.37 \mathrm{e}-5$ & 2.11 & 0.72 \\
\hline 1.2 & $3 e-7$ & $6.46 \mathrm{e}-4$ & $5.47 \mathrm{e}-4$ & 3.31 & 0.16 \\
\hline 1.2 & $5 e-7$ & $3.51 \mathrm{e}-4$ & $2.54 \mathrm{e}-4$ & 2.84 & 0.28 \\
\hline 1.2 & $7 e-7$ & $2.56 \mathrm{e}-4$ & $1.72 \mathrm{e}-4$ & 2.57 & 0.33 \\
\hline 1.2 & $9 e-7$ & $2.00 \mathrm{e}-4$ & $1.26 \mathrm{e}-4$ & 2.35 & 0.38 \\
\hline 1.2 & $2 e-6$ & $8.16 \mathrm{e}-5$ & $1.86 \mathrm{e}-5$ & 1.56 & 0.84 \\
\hline 1.3 & $3 e-7$ & $1.66 \mathrm{e}-4$ & $1.23 \mathrm{e}-4$ & 2.74 & 0.26 \\
\hline 1.3 & $5 e-7$ & $9.72 \mathrm{e}-5$ & $6.26 e-5$ & 2.28 & 0.38 \\
\hline 1.3 & $7 e-7$ & $6.60 e-5$ & $3.59 \mathrm{e}-5$ & 1.99 & 0.44 \\
\hline 1.3 & $9 e-7$ & $5.53 e-5$ & $2.72 \mathrm{e}-5$ & 1.81 & 0.49 \\
\hline 1.3 & $2 e-6$ & $2.21 \mathrm{e}-5$ & $1.95 \mathrm{e}-6$ & 1.14 & 0.95 \\
\hline 1.35 & $3 e-7$ & $4.80 e-5$ & $3.06 e-5$ & 2.21 & 0.35 \\
\hline 1.35 & $5 e-7$ & $2.70 e-5$ & $1.26 \mathrm{e}-5$ & 1.74 & 0.52 \\
\hline 1.35 & $7 e-7$ & $1.96 \mathrm{e}-5$ & $7.62 \mathrm{e}-6$ & 1.45 & 0.62 \\
\hline 1.35 & $9 e-7$ & $1.59 \mathrm{e}-5$ & $4.87 e-6$ & 1.26 & 0.70 \\
\hline 1.35 & $1.8 \mathrm{e}-6$ & $8.39 \mathrm{e}-6$ & $7.28 \mathrm{e}-8$ & 0.60 & 0.96 \\
\hline
\end{tabular}

the accretion phase is shorter since the higher mass-accretion rates (e.g., Prialnik et al.1982). Therefore, we can expect that the recurrent phenomena of He-shell flashes are more frequently to occur on massive ONe WDs with higher accretion rates. Hillman et al. (2016) investigate the He-accreting process of CO WDs, and suggested that the nova cycle duration decreases gradually if the $\mathrm{He}$-shell has experienced enough flashes. The reason is that the mass of WD is increased after multicycle flashes, and the WD is heated by the He-shell flashes, resulting in more frequent nova outbursts and weaker He-shell flashes. Thus, we speculate that He-accreting ONe WDs can increase their mass after experiencing He-shell flashes, which may lead to AIC events when their mass is close to $M_{\mathrm{Ch}}$, as studied in previous (e.g., Brooks et al. 2017a; Wu \& Wang 2018).

The ignition mass $M_{\mathrm{ign}}$ is defined as the minimum helium shell mass that can trigger dynamical burning. Panel (c) represents the He-shell ignition masses changing with ONe WD masses and massaccretion rates. From this figure, we can see that the ignition mass decreases with ONe WD mass and mass-accretion rate. This is because the critical pressure required for the ignition of He-shell is a function of ONe WD mass and ignition mass, in which ignition mass varies inversely with ONe WD mass (see Starrfield 1989). For a given ONe WD mass, a higher mass-accretion rate leads to a higher temperature growth rate of the accreted shell, resulting in a lower ignition mass (e.g., Prialnik et al. 1982). Tab. 1 displays the properties of He-shell flashes, including the ONe WD mass $M_{\mathrm{WD}}$, the mass-accretion rate $\dot{M}_{\text {acc }}$, the ignited mass $M_{\mathrm{ign}}$, the ejected mass $M_{\mathrm{ej}}$, the nova cycle duration $D$ and the mass-retention efficiency $\eta_{\mathrm{He}}$. From this table, we can infer that mass-retention efficiency decreases with the ignition mass for a given ONe WD mass. For example, in the case of a $1.35 M_{\odot} \mathrm{ONe} \mathrm{WD}$, there is almost no mass loss when the ignition mass is $8.39 \times 10^{-6} M_{\odot}$, but almost all accretion material is lost when the ignition mass is $4.80 \times 10^{-5} M_{\odot}$. If the ignition mass is smaller than a critical value, the mass-retention efficiency would be close to 1, which means that no mass loss would occur (see also Kato et al. 1989).

According to the results of Yaron et al. (2005), a nova burst occurs only when the cooling timescale ( $\left.\tau_{\text {cool }}\right)$ is longer than the accretion timescale $\left(\tau_{\text {acc }}\right)$. Obviously, the value of $\tau_{\text {acc }}$ is much lower for a higher accretion rate, resulting in weaker He-shell flashes and less ejected mass (see also Starrfield et al. 
Table 2: Mass fractions of nuclear products during a nova cycle.

\begin{tabular}{cccccccc}
\hline \hline & & & & & & \\
Set & $M_{\mathrm{WD}}\left(M_{\odot}\right)$ & $\dot{M}_{\mathrm{acc}}\left(M_{\left.\odot \mathrm{yr}^{-1}\right)}\right.$ & $\mathrm{C}$ & $\mathrm{O}$ & $\mathrm{Ne}$ & $\mathrm{Mg}$ & $\mathrm{Si}$ \\
\hline & 1.20 & $3 \mathrm{e}-7$ & $4.10 \mathrm{e}-01$ & $1.66 \mathrm{e}-01$ & $7.84 \mathrm{e}-02$ & $3.36 \mathrm{e}-01$ & $7.60 \mathrm{e}-03$ \\
1 & 1.20 & $5 \mathrm{e}-7$ & $4.42 \mathrm{e}-01$ & $1.95 \mathrm{e}-01$ & $8.65 \mathrm{e}-02$ & $2.73 \mathrm{e}-01$ & $6.00 \mathrm{e}-03$ \\
2 & 1.20 & $7 \mathrm{e}-7$ & $4.56 \mathrm{e}-01$ & $2.14 \mathrm{e}-01$ & $8.83 \mathrm{e}-02$ & $2.36 \mathrm{e}-01$ & $4.99 \mathrm{e}-03$ \\
3 & 1.20 & $9 \mathrm{e}-7$ & $4.55 \mathrm{e}-01$ & $2.53 \mathrm{e}-01$ & $9.07 \mathrm{e}-02$ & $1.96 \mathrm{e}-01$ & $4.60 \mathrm{e}-03$ \\
4 & 1.35 & $7 \mathrm{e}-7$ & $2.22 \mathrm{e}-01$ & $6.08 \mathrm{e}-03$ & $9.76 \mathrm{e}-03$ & $5.41 \mathrm{e}-01$ & $2.21 \mathrm{e}-01$ \\
5 & & & & &
\end{tabular}

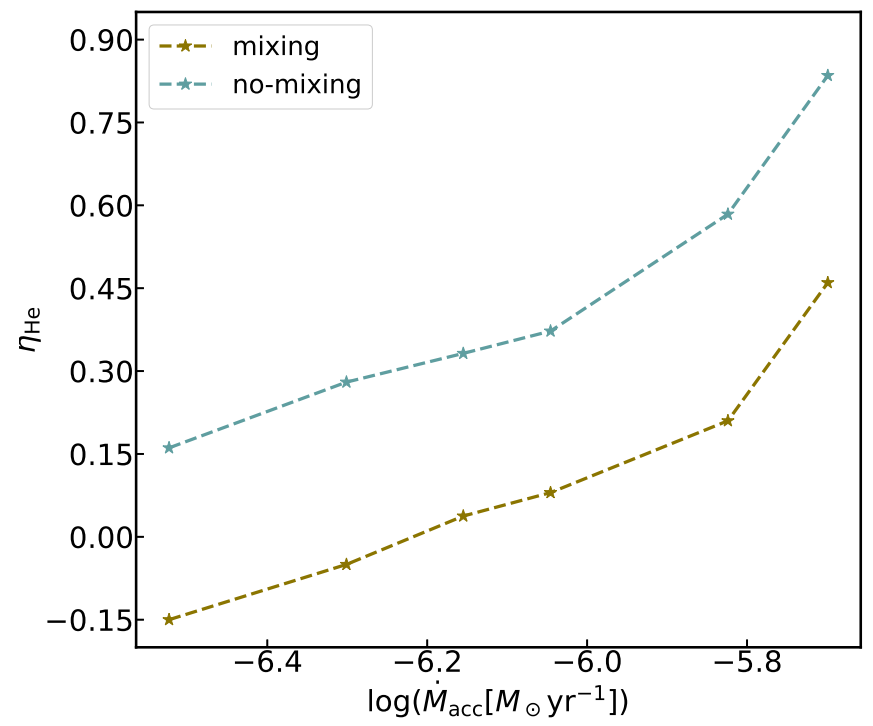

Fig. 4: Influence of mixing on the mass-retention efficiencies of a $1.2 M_{\odot}$ ONe WD.

2012). On the contrary, a lower accretion rate results in a stronger degenerate helium envelope since long cooling time. If the conditions of He-shell flashes can be reached by gradually accumulating Herich materials, a stronger burst will be triggered and blows away more material. However, we found that the temperature of He-shell drops quickly and it is hard to reach the conditions of He-shell flashes for very low accretion rates.

The chemical abundances (mass fraction) of nuclear products during a nova cycle are summarized in Tab. 2. It is apparent that the elements produced during the $\mathrm{He}$-shell flashes are sensitive to the $\mathrm{ONe}$ WDs masses and accretion rates. We found that $\mathrm{C}, \mathrm{O}$ and $\mathrm{Ne}$ increase with mass-accretion rates while $\mathrm{Mg}$ and $\mathrm{Si}$ decrease. This is because higher mass-accretion rates result in higher nuclear burning rates and weaker He-shell flashes. Thus, more He-rich material could be burn into $\mathrm{C}, \mathrm{O}$ and then Ne. On the other hand, as the peak temperature is higher for low mass-accretion rates, more heavier elements, such as $\mathrm{Mg}$ and $\mathrm{Si}$ are produced. In addition, $\mathrm{C}, \mathrm{O}$ and $\mathrm{Ne}$ decrease with WD masses while $\mathrm{Mg}$ and $\mathrm{Si}$ increase. This is due to the higher peak temperature caused by the stronger surface gravity acceleration for a massive WD. The trend of this result is consistent with Kato et al. (2018). 


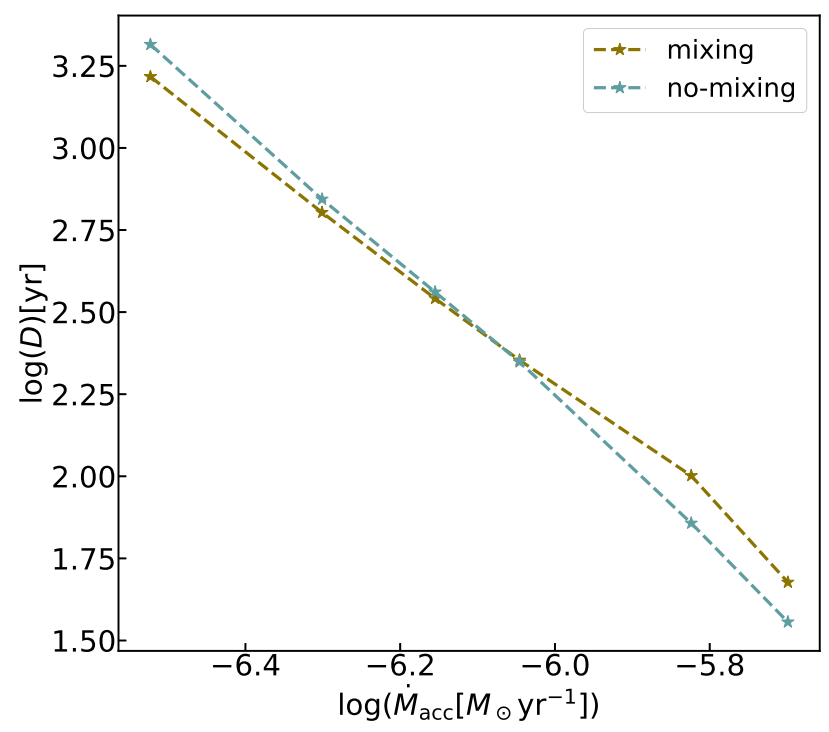

Fig. 5: Influence of mixing on the nova cycle durations of a 1.2 $M_{\odot}$ ONe WD.

Table 3: Properties of He-shell flashes with mixing, in which $M_{\mathrm{WD}}=1.2 M_{\odot}$.

\begin{tabular}{|c|c|c|c|c|}
\hline 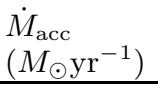 & $\begin{array}{c}M_{\text {ign }} \\
\left(M_{\odot}\right) \\
\end{array}$ & $\begin{array}{c}M_{\mathrm{ej}} \\
\left(M_{\odot}\right) \\
\end{array}$ & $\begin{array}{c}\log (D) \\
(\mathrm{yr}) \\
\end{array}$ & $\eta_{\mathrm{He}}$ \\
\hline $3 e-7$ & $6.20 \mathrm{e}-4$ & $4.91 \mathrm{e}-4$ & 3.22 & -0.15 \\
\hline $5 e-7$ & $3.49 \mathrm{e}-4$ & $2.50 \mathrm{e}-4$ & 2.80 & -0.05 \\
\hline $7 e-7$ & $2.44 \mathrm{e}-4$ & $1.14 \mathrm{e}-4$ & 2.54 & 0.04 \\
\hline $9 e-7$ & $2.06 \mathrm{e}-4$ & $8.51 \mathrm{e}-5$ & 2.35 & 0.08 \\
\hline $1.5 \mathrm{e}-6$ & $1.39 \mathrm{e}-4$ & $4.13 e-5$ & 2.02 & 0.21 \\
\hline $2 e-6$ & $9.27 e-5$ & $7.54 \mathrm{e}-6$ & 1.68 & 0.46 \\
\hline
\end{tabular}

\subsection{Influence of mixing process}

The mass-retention efficiencies provided in this work are likely the lower limit. We adopt a superEddington wind during the He-shell flashes, in which the material is assumed to be ejected if the escape speed is reached. In addition, if the rotation of ONe WD is considered, the He-shell flash would be much weaker since the weaker effective gravity and the material of WD core by rotational mixing at the base of the He-shell, which may lead to larger mass-retention efficiencies (see Yoon et al. 2004). Moreover, the mass of ONe WD can exceed $M_{\mathrm{Ch}}$ when the rotation effects are taken into account (e.g., Yoon \& Langer 2004; Chen \& Li 2009; Justham 2011; Hachisu et al. 2012; Wang et al. 2014).

A number of Ne enriched novae have been observed, e.g., V693 CrA 1981, V1370 Aql 1982, QU Vul 1984, V1668 Cyg 1978, V838 Her 1991, 1974 Cyg 1992 (e.g., Gehrz et al. 1998; Wanajo et al. 1999; Downen et al. 2013). An important feature of Ne novae is the significant enrichments of O, Ne, $\mathrm{Mg}$ and $\mathrm{Al}$ detected in the ejection (e.g., Andrea et al. 1994; José \& Hernanz 1998; Gehrz et al. 1998; Wanajo et al. 1999). José \& Hernanz (1998) investigated the elemental abundance in the ejection of $\mathrm{Ne}$ novae, and found that the considered mixing model may be a suitable way to explain the observed ele- 


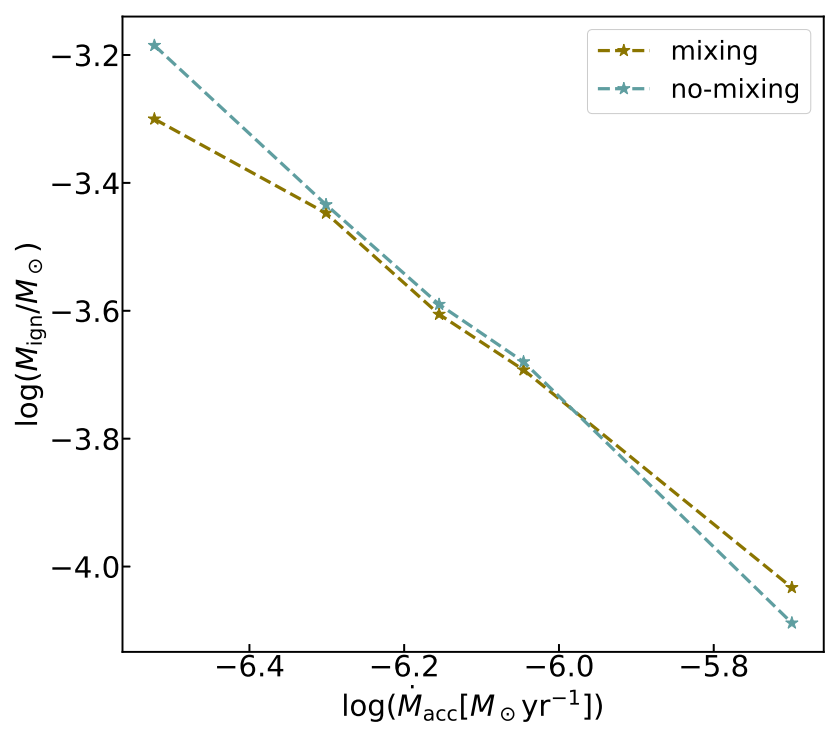

Fig. 6: Influence of mixing on the ignition masses of a $1.2 M_{\odot}$ ONe WD.

ment abundance. During the mass-accretion process, the accreted envelope may be mixed with the material in the underlying WD core. The efficiency of mixing on accreting WDs depends on which diffusion physics are included, e.g., if magnetic torques are included, mixing during accretion tends to be minimal (e.g., Yoon \& Langer 2004; Neunteufel et al. 2017). However, the mixing mechanism is not completely clear. There are some hypotheses for the mixing mechanism, such as diffusion, shear mixing, convective overshooting and Kelvin-Helmholtz instabilities (e.g., Prialnik \& Kovetz 1984; Kutter \& Sparks 1987; Iben et al. 1991; Glasner et al. 1997; Kercek et al. 1998; Casanova et al. 2016).

Here, we provide a simplified mixing case without including the physical mechanism of the mixing process, as described in Politano et al. (1995). We assume that the accreted material from the companion is mixed by a given element fraction of the underlying $\mathrm{ONe} W D$ core. We set the mixing fraction to be 0.5 , which has been suggested as a representative value (e.g., Livio \& Truran 1994). In Tab. 3, we provide the properties of He-shell flashes for the mixing cases. Fig. 4 shows the influence of mixing on the mass-retention efficiencies of a 1.2 $M_{\odot}$ ONe WD. It is obvious that the mass-retention efficiency in the mixing case is lower than that without considering mixing for a given mass-accretion rate. Especially for the cases with low mass-accretion rates (e.g., $\dot{M}_{\mathrm{acc}}=3 \times 10^{-7} M_{\odot} \mathrm{yr}^{-1}$ ), the value of $\eta_{\mathrm{He}}$ is negative, which means that the ONe WD mass is decreasing. This is because the core material is dredged up from the underlying ONe WD during mixing process, and thus ONe WD is losing mass as a result of continued outbursts. Meanwhile, even in the case of higher accretion rates, the mass of ONe WD under mixing conditions is more difficult to grow than the cases without mixing. This result indicates that the consideration of the mixing process may also affect the predicted birthrates of AIC events. Therefore, we suggest that the mixing process is important for the study of the final fate of He-accreting ONe WDs. In addition, Figs 5-6 present that the influence of mixing on the nova cycle duration and ignition mass of a $1.2 M_{\odot}$ ONe WD. These two figures indicate that the mixing process has slight influence on the nova cycle duration and ignition mass. This is because the nova cycle duration and ignition mass are more sensitive to the temperature and density of the accreted material, but not very sensitive to their composition. 


\section{DISCUSSION AND CONCLUSIONS}

It has been suggested that AIC events are about 5 mag fainter than typical normal $\mathrm{SNe}$ Ia, and can only last from a few days to a week, so that AIC is difficult to be identified in the observations. According to Piro \& Kulkarni (2013), radio sources that last for a few months may be caused by AIC events. In addition, it is possible that a small amount of ${ }^{56} \mathrm{Ni}$ is synthesized in the AIC process (e.g., Dessart et al. 2006; Darbha et al. 2010). These characteristics may be used to identify AIC events. Tab. 2 shows that more $\mathrm{Mg}$ and $\mathrm{Si}$ can be produced in the He-shell flashes for massive ONe WDs. We expect that the surface of the WDs would be covered with a $\mathrm{Mg} / \mathrm{Si}$-rich shell if the WDs masses increase to approach $M_{\mathrm{Ch}}$ by accreting He-rich material. Therefore, the enrichments of $\mathrm{Mg} / \mathrm{Si}$ may be detectable in AIC events formed by ONe WDs accreting He-rich material.

In addition, it is difficult to accurately distinguish whether the accretor is a CO WD or an ONe WD if the accretion rate is significantly large, as there is no obvious mixing process or significant enrichment elemental abundance of WD in the ejection. As mentioned above, V445 Pup is the only He nova that has been identified so far. The WD mass is suggested to be $\geq 1.35 M_{\odot}$ and half of the accreted material could remain on its surface (see Kato et al. 2008). Kato et al. (2008) suggested that the WD of V445 Pup is a CO WD but not an ONe WD since no signatures of neon enhancement were detected, and argued that V445 Pup is a strong candidate of SNe Ia (see also Woudt \& Steeghs 2005; Wang 2018a). However, the carbon features originated from WD core or helium burning are still not determined, and it is still unclear whether there is a mixing process or not during mass-accreting process (e.g., Kato \& Hachisu 2004). Further detailed researches are needed in future studies to provide more precise nature of the WD in V445 Pup.

By using the stellar evolution code MESA, we investigated the long-term evolution of accreting ONe WDs that undergo multicycle He-shell flashes with initial masses ranging from 1.1 to $1.35 M_{\odot}$ and various accretion rates in the range of $3 \times 10^{-7}-2 \times 10^{-6} M_{\odot} \mathrm{yr}^{-1}$. We found that the massretention efficiency both increases with the ONe WD mass and the mass-accretion rate. More massive ONe WDs or higher mass-accretion rates lead to shorter nova cycle durations, which indicates that the ONe WD mass grows faster for the cases with more massive initial ONe WDs and higher mass-accretion rates. Meanwhile, we also found that the ignition mass both decreases with the ONe WD mass and the mass-accretion rate. We also present the nuclear products in different accretion scenarios. In addition, for a given ONe WD mass and mass-accretion rate, the mass-retention efficiency would be lower after considering the mixing effects. In order to put more constraints on the process of He-shell flashes, we hope that more He novae and Ne novae can be identified in the observations.

Acknowledgements We thank the anonymous referee for valuable comments that can help to improve the paper. BW is supported by the National Natural Science Foundation of China (Nos 11873085, 11673059 and 11521303), the Chinese Academy of Sciences (No QYZDB-SSW-SYS001), and the Yunnan Province (Nos 2018FB005 and 2019FJ001). DDL is supported by the National Natural Science Foundation of China (No. 11903075) and the Western Light Youth Project of Chinese Academy of Sciences.

\section{References}

Abdikamalov, E. B., Ott, C. D., Rezzolla, L., et al. 2010, Phys. Rev. D, 81, 044012

Ablimit, I., \& Li, X.-D. 2015, ApJ, 800, 98

Andrea, J., Drechsel, H., \& Starrfield, S. 1994, A\&A, 291, 869

Bailyn, C. D., \& Grindlay, J. E. 1990, ApJ, 353, 159

Bhattacharya, D., \& van den Heuvel, E. P. J. 1991, Phys. Rep., 203, 1

Brooks, J., Bildsten, L., Marchant, P., \& Paxton, B. 2015, ApJ, 807, 74

Brooks, J., Bildsten, L., Schwab, J., \& Paxton, B. 2016, ApJ, 821, 28

Brooks, J., Schwab, J., Bildsten, L., Quataert, E., \& Paxton, B. 2017a, ApJ, 843, 151

Brooks, J., Schwab, J., Bildsten, L., Quataert, E., \& Paxton, B. 2017b, ApJ, 834, L9

Canal, R., Garcia, D., Isern, J., \& Labay, J. 1990, ApJ, 356, L51 
Casanova, J., José, J., García-Berro, E., \& Shore, S. N. 2016, A\&A, 595, A28

Chen, H.-L., Woods, T. E., Yungelson, L. R., et al. 2019, MNRAS, 490, 1678

Chen, W. C., \& Li, X.-D. 2009, ApJ, 702, 686

Chen, W. C., \& Liu, W. M. 2013, MNRAS, 432, L75

Dar, A., Kozlovsky, B. Z., Nussinov, S., \& Ramaty, R. 1992, ApJ, 388, 164

Darbha, S., Metzger, B. D., Quataert, E., et al. 2010, MNRAS, 409, 846

Dessart, L., Burrows, A., Ott, C. D., et al. 2006, ApJ, 644, 1063

Downen, L. N., Iliadis, C., José, J., \& Starrfield, S. 2013, ApJ, 762, 105

Faulkner, A. J., Stairs, I. H., Kramer, M., et al. 2004, MNRAS, 355, 147

Fryer, C., Benz, W., Herant, M., \& Colgate, S. A. 1999, ApJ, 516, 892

Gehrz, R. D., Truran, J. W., Williams, R. E., \& Starrfield, S. 1998, PASP, 110, 3

Geier, S., Nesslinger, S., Heber, U., et al. 2007, A\&A, 464, 299

Glasner, S. A., Livne, E., \& Truran, J. W. 1997, ApJ, 475, 754

Hachisu, I., Kato, M., Saio, H., \& Nomoto, K. 2012, ApJ, 744, 69

Hillebrandt, W., \& Niemeyer, J. C. 2000, ARA\&A, 38, 191

Hillman, Y., Prialnik, D., Kovetz, A., \& Shara, M. M. 2015, MNRAS, 446, 1924

Hillman, Y., Prialnik, D., Kovetz, A., \& Shara, M. M. 2016, ApJ, 819, 168

Iben, Icko, J., Fujimoto, M. Y., \& MacDonald, J. 1991, ApJ, 375, L27

Iglesias, C. A., \& Rogers, F. J. 1996, ApJ, 464, 943

José, J., \& Hernanz, M. 1998, ApJ, 494, 680

Justham, S. 2011, ApJ, 730, L34

Kato, M., \& Hachisu, I. 1994, ApJ, 437, 802

Kato, M., \& Hachisu, I. 1999, ApJ, 513, L41

Kato, M., \& Hachisu, I. 2004, ApJ, 613, L129

Kato, M., Hachisu, I., Kiyota, S., \& Saio, H. 2008, ApJ, 684, 1366

Kato, M., Saio, H., \& Hachisu, I. 1989, ApJ, 340, 509

Kato, M., Saio, H., \& Hachisu, I. 2018, ApJ, 863, 125

Kato, T., Kanatsu, K., Takamizawa, K., Takao, A., \& Stubbings, R. 2000, IAU Circ., 7552, 1

Kercek, A., Hillebrandt, W., \& Truran, J. W. 1998, A\&A, 337, 379

Kutter, G. S., \& Sparks, W. M. 1987, ApJ, 321, 386

Liu, D., \& Wang, B. 2020, MNRAS, 494, 3422

Liu, D., Wang, B., Chen, W., Zuo, Z., \& Han, Z. 2018, MNRAS, 477, 384

Liu, D., Zhou, W.-H., Wu, C.-Y., \& Wang, B. 2015, Research in Astronomy and Astrophysics, 15, 1813

Liu, W.-M., \& Li, X.-D. 2017, ApJ, 851, 58

Livio, M., \& Truran, J. W. 1994, ApJ, 425, 797

Lyutikov, M., \& Toonen, S. 2017, arXiv e-prints, arXiv:1709.02221

Miyaji, S., Nomoto, K., Yokoi, K., \& Sugimoto, D. 1980, PASJ, 32, 303

Moriya, T. J. 2016, ApJ, 830, L38

Nelemans, G., Portegies Zwart, S. F., Verbunt, F., \& Yungelson, L. R. 2001, A\&A, 368, 939

Neunteufel, P., Yoon, S. C., \& Langer, N. 2017, A\&A, 602, A55

Nomoto, K. 1982, ApJ, 253, 798

Nomoto, K. 1984, ApJ, 277, 791

Nomoto, K., \& Iben, I., J. 1985, ApJ, 297, 531

Nomoto, K., Iwamoto, K., \& Kishimoto, N. 1997, Science, 276, 1378

Nomoto, K., \& Kondo, Y. 1991, ApJ, 367, L19

Paxton, B., Bildsten, L., Dotter, A., et al. 2011, ApJS, 192, 3

Paxton, B., Cantiello, M., Arras, P., et al. 2013, ApJS, 208, 4

Paxton, B., Marchant, P., Schwab, J., et al. 2015, ApJS, 220, 15

Paxton, B., Schwab, J., Bauer, E. B., et al. 2018, ApJS, 234, 34

Piersanti, L., Tornambé, A., \& Yungelson, L. R. 2014, MNRAS, 445, 3239

Piro, A. L., \& Kollmeier, J. A. 2016, ApJ, 826, 97

Piro, A. L., \& Kulkarni, S. R. 2013, ApJ, 762, L17 
Podsiadlowski, P., Rappaport, S., \& Pfahl, E. D. 2002, ApJ, 565, 1107

Politano, M., Starrfield, S., Truran, J. W., Weiss, A., \& Sparks, W. M. 1995, ApJ, 448, 807

Prialnik, D., \& Kovetz, A. 1984, ApJ, 281, 367

Prialnik, D., Livio, M., Shaviv, G., \& Kovetz, A. 1982, ApJ, 257, 312

Qian, Y. Z., \& Wasserburg, G. J. 2007, Phys. Rep., 442, 237

Saio, H., \& Nomoto, K. 2004, ApJ, 615, 444

Schwab, J., Bildsten, L., \& Quataert, E. 2017, MNRAS, 472, 3390

Schwab, J., Quataert, E., \& Bildsten, L. 2015, MNRAS, 453, 1910

Schwab, J., Quataert, E., \& Kasen, D. 2016, MNRAS, 463, 3461

Shen, K. J., \& Bildsten, L. 2009, ApJ, 699, 1365

Starrfield, S. 1989, in Classical Novae, 39

Starrfield, S., Iliadis, C., Timmes, F. X., et al. 2012, Bulletin of the Astronomical Society of India, 40, 419

Tauris, T. M., Sanyal, D., Yoon, S. C., \& Langer, N. 2013, A\&A, 558, A39

Usov, V. V. 1992, Nature, 357, 472

Wanajo, S., Hashimoto, M.-a., \& Nomoto, K. 1999, ApJ, 523, 409

Wang, B. 2018a, Research in Astronomy and Astrophysics, 18, 049

Wang, B. 2018b, MNRAS, 481, 439

Wang, B., \& Han, Z.-W. 2010, Research in Astronomy and Astrophysics, 10, 681

Wang, B., Justham, S., Liu, Z. W., et al. 2014, MNRAS, 445, 2340

Wang, B., Li, Y., Ma, X., et al. 2015, A\&A, 584, A37

Wang, B., \& Liu, D. 2020, arXiv e-prints, arXiv:2005.01880

Wang, B., Podsiadlowski, P., \& Han, Z. 2017, MNRAS, 472, 1593

Wheeler, J. C., Cowan, J. J., \& Hillebrandt, W. 1998, ApJ, 493, L101

Woosley, S. E., \& Baron, E. 1992, ApJ, 391, 228

Woudt, P. A., \& Steeghs, D. 2005, Astronomical Society of the Pacific Conference Series, Vol. 330, Spectroscopy and Near-Infrared Photometry of the Helium Nova V445 Puppis, ed. J. M. Hameury \& J. P. Lasota, Astronomical Society of the Pacific Conference Series, Vol. 330, The Astrophysics of Cataclysmic Variables and Related Objects, ed. J. M. Hameury \& J. P. Lasota, 451

Woudt, P. A., Steeghs, D., Karovska, M., et al. 2009, ApJ, 706, 738

Wu, C., \& Wang, B. 2018, Research in Astronomy and Astrophysics, 18, 036

Wu, C., Wang, B., Liu, D., \& Han, Z. 2017, A\&A, 604, A31

Yaron, O., Prialnik, D., Shara, M. M., \& Kovetz, A. 2005, ApJ, 623, 398

Yoon, S. C., \& Langer, N. 2004, A\&A, 419, 623

Yoon, S. C., Langer, N., \& Scheithauer, S. 2004, A\&A, 425, 217

Zha, S., Leung, S.-C., Suzuki, T., \& Nomoto, K. 2019, ApJ, 886, 22 